\title{
Unary-Coded Dimming Control Improves ON-OFF Keying Visible Light Communication
}

\author{
Zunaira Babar, Mohd Azri Mohd Izhar, Hung Viet Nguyen, Panagiotis Botsinis, \\ Dimitrios Alanis, Daryus Chandra, Soon Xin Ng, Robert G. Maunder and Lajos Hanzo
}

\begin{abstract}
An ideal Visible Light Communication (VLC) system should facilitate reliable data transmission at high throughputs, while also providing flicker-free illumination at the user-defined dimming level. In this spirit, we conceive a unary code aided dimming scheme for On-Off Keying (OOK) modulated VLC systems. The proposed unary-coded scheme facilitates joint dimming and throughput control, while relying on iterative decoding. It is demonstrated that the proposed unary-coded dimming scheme provides attractive throughput gains over its contemporaries and it is also capable of approaching the theoretical throughput limit. Furthermore, we design novel joint dimming-Forward Error Correction (FEC) coding schemes, which significantly outperform their compensation time dimming based counterparts in terms of the attainable Bit Error Rate (BER) performance as well as the throughput. Finally, in the quest for approaching the capacity, we also optimize our system using EXtrinsic Information Transfer (EXIT) charts and demonstrate an SNR-gain of upto $6 \mathrm{~dB}$ over the compensation time dimming based classic benchmarker.
\end{abstract}

Keywords-Visible Light Communication, Dimming control, Iterative Decoding, EXIT charts.

\section{INTRODUCTION}

Visible Light Communication (VLC) [1]-[4] has emerged as a potential complement to the classic Radio Frequency (RF) based communication, particularly in short range indoor applications. In contrast to RF systems, which rely on the scarce RF spectrum, VLC harnesses the abundant licensefree visible light band (400 to $800 \mathrm{THz}$ ) for supporting both illumination as well as communication. This is achieved by exploiting the already available Light Emitting Diode (LED) based lighting infrastructure for wireless data transmission. Explicitly, data may be transmitted by intensity based modulation of the available light sources, for example LEDs and laser diodes, at On/Off rates beyond the human eye's fusion

Z. Babar, H. V. Nguyen, P. Botsinis, D. Alanis, D. Chandra, S. X. Ng, R. G. Maunder and L. Hanzo are with the School of Electronics and Computer Science, University of Southampton, SO17 1BJ, United Kingdom. Email: \{zb2g10, hvn08r, pb1y14, da1d16, dc2n14, sxn, rm, lh\}@ecs.soton.ac.uk.

M. A. M. Izhar is with the Wireless Communication Center and UTM Razak School of Engineering and Advanced Technology, Universiti Teknologi Malaysia, Jalan Sultan Yahya Petra, Kuala Lumpur, 54100, Malaysia. Email: mohdazri.kl@utm.my.

The financial support of the European Research Council under the Advanced Fellow Grant, that of the Royal Society's Wolfson Research Merit Award and that of the Engineering and Physical Sciences Research Council under Grant EP/L018659/1 is gratefully acknowledged. The use of the IRIDIS High Performance Computing Facility at the University of Southampton is also acknowledged. The research data for this paper is available at [http://doi.org/10.5258/SOTON/D0269].

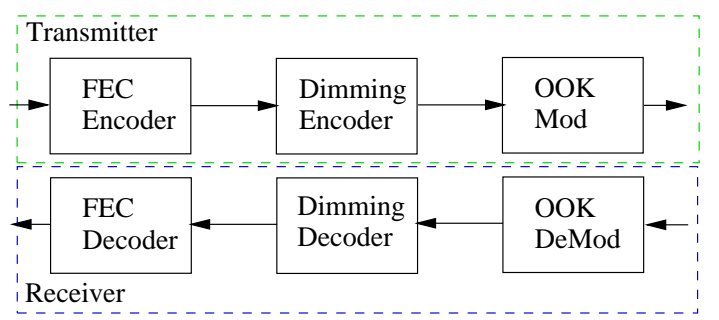

Fig. 1: Schematic of a VLC system having dimming control functionality.

frequency. Consequently, VLC technology may be readily integrated into existing lighting systems with the aid of simple and inexpensive off-the-shelf components.

Since a VLC system serves the dual function of illumination and communication, it should be capable of facilitating dimming control, while also providing high throughputs (or data transmission rates). Dimming control is particularly important for indoor applications, where a user may want to maintain a desired lighting ambiance. The interest in dimming control functionality has further increased in the wake of everincreasing global energy consumption, which is expected to rise further by another $60 \%$ over the next 25 years [5].

Fig. 1 shows the general schematic of an On-Off Keying (OOK) modulated VLC system, which relies on a serial concatenation of Forward Error Correction (FEC) and dimming modules for achieving error correction and dimming control, respectively. In the simplest VLC systems, the dimming encoder adopts compensation time dimming [1], where 0 or 1valued compensation symbols are inserted in the FEC encoded data frame for achieving the desired dimming level. At the receiver, the so-called 'Dimming decoder' throws away the compensation symbols for the sake of recovering the received encoded data frame, which is then fed to the FEC decoder for error correction. Since compensation symbols merely act as fillers for achieving the desire dimming level and do not carry any useful information, this dimming approach severely degrades the resultant throughput. For the sake of alleviating this throughput degradation, an Inverse Source Coding (ISC) scheme was used for achieving dimming control in [6]. ISC is capable of approaching the theoretical throughput limit. However, ISC is a theoretical framework for reliable systems, which may degrade the system's Bit Error Rate (BER) performance, when used in conjunction with an FEC code 
for communicating over noisy channels. In pursuit of jointly controlling both the dimming and throughput, variable-rate Multipulse Pulse Position Modulation (MPPM) aided dimming was developed in [7], [8], which is capable of approaching the information theoretic throughput limit as the codeword length is increased. However, a long codeword increases the associated encoding/decoding complexity and imposes higher storage requirements. In this context, efficient MPPM encoding and hard-decision based decoding implementations were conceived in [8]. However, soft decoding of MPPM still relies on a Look-Up Table (LUT).

The aforementioned dimming control schemes must be used in conjunction with FEC codes for mitigating the deleterious effects of channel noise. Unfortunately, the coded MPPM schemes [9]-[11] conceived for free-space optical communications are not suitable for VLC systems, where long codeword lengths, and hence large LUTs, are required for maximizing the achievable throughput at the desired dimming level. For the sake of achieving joint dimming and throughput control as well as error correction, optimized constant-weight codes were conceived in [12]. Prior to this, coding-based dimming schemes were developed in $[13]-[16]$, which relied on compensation time dimming.

Inspired by the joint decoding and detection based concatenated designs of [3], [4], [17]-[19], which invoke iterative decoding, in this paper we conceive new iterative dimmingFEC schemes. Explicitly, our main contributions are:

- We propose a unary-coded dimming control scheme in Section [I] which is capable of achieving a higher throughput than the compensation time dimming as well as the MPPM-based scheme. More specifically, the throughput of our unary-coded scheme tends to approach the theoretical limit faster, as we move away from the $50 \%$ dimming level. Furthermore, we demonstrate that a unary code is capable of ensuring a flicker-free dimming level.

- In contrast to the MPPM-based scheme, a unary code has a low encoding complexity and facilitates soft trellis decoding. Hence, we conceive a joint unary-FEC code design in Section [II] which is capable of exchanging extrinsic information between the dimming and FEC modules of the receiver using iterative decoding. To the best of authors' knowledge, this is the first instantiation of iterative dimming-FEC decoding schemes.

- We quantify the unary-constrained capacity over an Additive White Gaussian Noise (AWGN) channel using EXtrinsic Information Transfer (EXIT) charts [17], [20] in Section IV The proposed unary-coded schemes incur a lower capacity loss, than the compensation time dimming.

- We demonstrate in Section $\nabla$ that the proposed unaryFEC designs provide attractive performance gains over their compensation time based counterparts. Quantitatively, our designs require about 3 to $6 \mathrm{~dB}$ lower $S N R$ for achieving a BER of $10^{-5}$ at dimming levels of $60 \%$ and $78 \%$, while also offering a 1.5 times higher throughput at the dimming level of $78 \%$. Furthermore, our designs operate arbitrarily close to the unary-constrained capacity, when the IRregular Convolutional Code (IRCC) of [21] is invoked.

\section{Dimming Control Methods}

\section{A. Compensation Time Dimming}

From the perspective of implementation, OOK is the simplest modulation method for a VLC system. Explicitly, OOK encodes 1-valued and 0 -valued data bits using On and Off pulses, respectively. When the data bits are equiprobable, OOK modulation yields a dimming level (also called brightness) of $50 \%$. This may be varied by changing the LED drive current, which alters the signal's On and Off levels. However, altering the current may lead to chromaticity shifts. Furthermore, a low drive current also reduces the transmission range. Alternatively, the dimming level can be adjusted by multiplexing the data and compensation symbols in time [1], [6], which is known as 'compensation time dimming'. Explicitly, 1-valued (On) or 0 -valued (Off) compensation symbols are multiplexed with the data symbols for achieving the arbitrary dimming level set by the usen. Unfortunately, this adversely affects the system's transmission efficiency, as it now exhibits a lower throughput. For example, a dimming level of $75 \%$ can be achieved for an $N$-bit data frame, if $N$ 1-valued (On) compensation symbols are added to the transmission frame, while $N 0$-valued compensation symbols are required for a dimming level of $25 \%$. In both these instances, the system throughput is reduced to half. Explicitly, the resultant normalized throughput $R_{\mathrm{CT}}$ is related to the desired dimming level $\gamma$ as follows:

$$
R_{\mathrm{CT}}=\left\{\begin{array}{ll}
2-2 \gamma, & \gamma>0.5 \\
2 \gamma, & \gamma \leq 0.5
\end{array} .\right.
$$

\section{B. Inverse Source Coding}

Let us recall that source coding is a data compression scheme, which takes a biased input, i.e. input having nonequiprobable bits or symbols, to generate equiprobable 0 and 1-valued bits, hence maximizing the source entropy. By contrast, dimming control may be viewed as a specific inverse operation of a source encoder, since it processes equiprobable bits to produce non-equiprobable bit values, so that we have $p_{1}=\gamma$ and $p_{0}=(1-\gamma)$, where $p_{1}$ and $p_{0}$ are the probabilities of having bit 1 and bit 0 , respectively. Consequently, the inverse of a source encoder may be used for achieving dimming control, hence it was termed as Inverse Source Coding (ISC) [6]. The resultant maximum achievable

\footnotetext{
${ }^{1}$ Compensation symbols may be appended either after each data frame or between every symbol. The former approach is generally termed as 'compensation time dimming' [1], while the latter is known as VariableOOK (VOOK) [7]. More specifically, in the VOOK scheme, dimming is controlled by varying the data duty cycle of the OOK signal and filling the inactive portions of the duty cycle with the 1-valued or 0 -valued compensation symbols.
} 
normalized throughput $R_{\mathrm{ISC}}$ is equivalent to the source entropy. Explicitly, we have:

$$
R_{\mathrm{ISC}}=-\gamma \log _{2}(\gamma)-(1-\gamma) \log _{2}(1-\gamma),
$$

which is the information theoretic throughput limit for a given dimming value $\gamma$. Potentially, any source code may be used as an ISC as long as its symbol probabilities may be adjusted according to the required dimming level. However, the number of bits in the decoded output of ISC may be higher (bit insertion) or lower (bit deletion) than the bits at the input of the ISC encoder, hence posing potential synchronization issues for the classic channel coding techniques.

\section{Multipulse Pulse Position Modulation}

In an $(n, w)$ Multipulse Pulse Position Modulation (MPPM) scheme, each symbol period is partitioned into $n$ slots so that the transmitter sends $1 \leq w \leq n$ optical signals during each symbol period. This results in $\left(\begin{array}{c}n \\ w\end{array}\right)$ unique symbols, which hence can encode $k=\left[\log _{2}\left(\begin{array}{c}n \\ w\end{array}\right)\right]$ data bits, where [.] denotes the decimation operator, which decimates the value to the nearest integer. From the perspective of dimming control in an OOK-assisted VLC system, an $(n, w)$ MPPM may be considered a constant-weight code having a weight $w$, which maps $k$ data bits onto an $n$-bit codeword so that the resulting codewords have $w 1$-valued bits. This yields a dimming level of $\gamma=w / n$ and a normalized throughput of $\left[\log _{2}\left(\begin{array}{l}n \\ w\end{array}\right)\right] / n$. Hence, the dimming level of an MPPM-assisted system can be controlled by changing the value of $w$, which also alters the system throughput.

The maximum normalized throughputs achieved by the aforementioned dimming control schemes, namely ISC, compensation time dimming (CT) and MPPM, are compared in Fig. 2. Recall that the ISC scheme sets the information theoretic throughput limit for a given dimming level $\gamma$. We may notice in Fig. 2 that the MPPM scheme approaches the ISC throughput as the codeword length $n \rightarrow \infty$. Unfortunately, the cardinality of the MPPM-based constant-weight code also increases drastically with $n$, as demonstrated in Fig. 3. Since soft decoding of MPPM relies on an LUT, it is not feasible to carry out soft decoding for high codeword lengths.

\section{Unary-Coded Dimming Control}

Unary codes constitute variable length source coding techniques [22], [23]. They map a symbol $i$ onto an $i$-bit codeword, which has $(i-1) 1$ 's followed by a zero (or equivalently $(i-1)$ 0's followed by a one). Table $\Pi$ enlists the first eleven codewords of the unary code. We may notice in Table I that the number of 1's (or equivalently 0 's) in the unary codeword increases with $i$. Explicitly, as we go down in Table I the dimming level of the codeword increases (or equivalently decreases). Inspired by this systematic change in the dimming level of the codewords, we propose to exploit unary codes for dimming control in VLC systems.

Let us consider a unary-coded dimming control scheme, which processes a $k$-bit equiprobable input to generate an $n$-bit

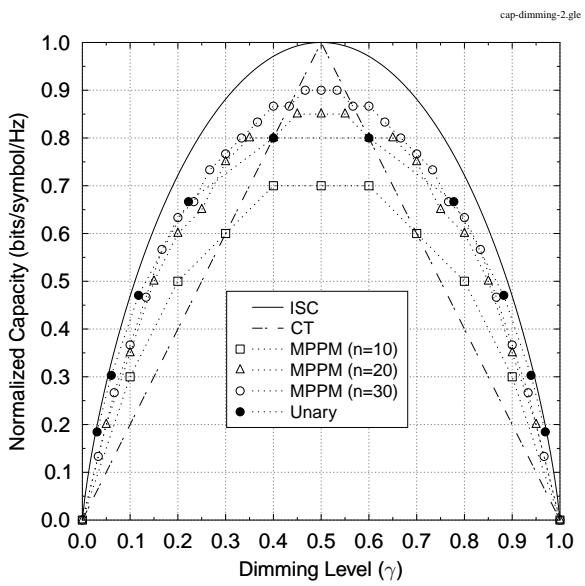

Fig. 2: Maximum normalized throughput achieved by the various dimming control methods, namely Inverse Source Coding (ISC), compensation time dimming (CT), Mulitpulse Pulse Position Modulation (MPPM) and the proposed unarycoded scheme.

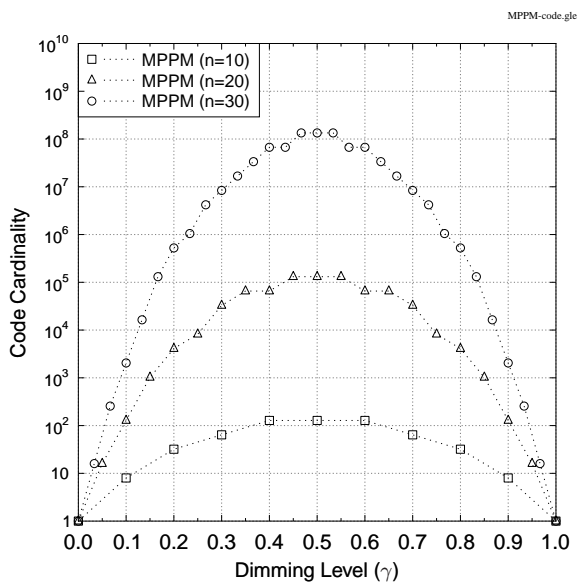

Fig. 3: Cardinality of an MPPM-based code parametrized by codeword lengths of $n=10,20$ and 30 .

codeword, where $n$ is the average codeword length, because a unary code produces variable length codewords. Explicitly, we have:

$$
n=\sum_{i \in \chi} P(i) i
$$

where $\chi=\{1,2,3, \ldots|\chi|\}$ constitutes the set of symbols and $P(i)=1 /|\chi|$. Furthermore, since we have $k$ data bits, the required cardinality of the code is $|\chi|=2^{k}$. Consequently, the unary code for our proposed dimming scheme is based on the first $2^{k}$ codewords of Table I, which is hence referred to as a $2^{k}$-level unary code. Based on this, Eq. (3) can be reformulated 


\begin{tabular}{ll}
\hline$i$ & Codeword \\
\hline 1 & 0 \\
2 & 10 \\
3 & 110 \\
4 & 1110 \\
5 & 11110 \\
6 & 111110 \\
7 & 111110 \\
8 & 1111110 \\
9 & 11111110 \\
10 & 111111110 \\
11 & 1111111110 \\
$\vdots$ & $\vdots$ \\
\hline
\end{tabular}

TABLE I: Codewords of the unary code.

\begin{tabular}{llll}
\hline $\mathrm{i}$ & $\begin{array}{l}\text { Input } \\
k \text {-bits }\end{array}$ & $\begin{array}{l}\text { Symbol } \\
\text { Probability }\end{array}$ & Codeword \\
\hline 1 & 000 & 0.125 & 0 \\
2 & 001 & 0.125 & 10 \\
3 & 010 & 0.125 & 110 \\
4 & 011 & 0.125 & 1110 \\
5 & 100 & 0.125 & 11110 \\
6 & 101 & 0.125 & 111110 \\
7 & 110 & 0.125 & 1111110 \\
8 & 111 & 0.125 & 11111110 \\
\hline
\end{tabular}

TABLE II: 8-level unary code $\left(\gamma_{u}=0.78\right.$ and $\left.R_{u}=0.667\right)$.

as:

$$
n=\frac{1}{2^{k}} \sum_{i=1}^{2^{k}} i .
$$

The resultant average dimming level $\gamma_{\mathrm{u}}$ may be computed as follows:

$$
\gamma_{\mathbf{u}}=\frac{\sum_{i=1}^{2^{k}-1} i}{\sum_{i=1}^{2^{k}} i},
$$

while the associated normalized throughput $R_{\mathrm{u}}$ is given by:

$$
R_{\mathrm{u}}=\frac{k}{n}=\frac{k 2^{k}}{\sum_{i=1}^{2^{k}} i} .
$$

As an example, let us consider the 8-level unary code of Table II which yields a dimming level of $\left(\frac{28}{36}=0.78\right)$ and a normalized throughput of $\left(\frac{3}{4.5}=0.667\right)$. The achievable dimming level and throughput of the unary-coded scheme can be controlled by varying the number of input bits $k$, as demonstrated in Table $\amalg$ and Fig. 2 Please note that dimming values of $\left(1-\gamma_{u}\right)$ can be obtained by using complementary unary mapping, which maps a symbol $i$ onto a codeword having $(i-1) 0$ 's followed by a one. We may observe in Fig. 2 that the throughput of our unary-coded scheme approaches that of the ISC, as we move further away from the dimming level of $50 \%$. The unary scheme outperforms the compensation time dimming approach in terms of its achievable throughput for $\gamma<0.4$ and $\gamma>0.6$. We may further observe in Fig. 2 that

\begin{tabular}{llll}
\hline$k$ & $|\chi|$ & $\gamma_{u}$ & $R_{u}$ \\
\hline 2 & 4 & 0.6 & 0.8 \\
3 & 8 & 0.78 & 0.667 \\
4 & 16 & 0.8824 & 0.4706 \\
5 & 32 & 0.9394 & 0.303 \\
6 & 64 & 0.9692 & 0.1846 \\
\hline
\end{tabular}

TABLE III: Unary-coded dimming control schemes.

the throughput of MPPM exceeds that of the unary code, as the codeword length is increased. However, soft-decoding of MPPM is not feasible for such long codewords, as illustrated previously in Fig. 3 By contrast, the unary code can be decoded using a symbol-level trellis [24], which is widely invoked for soft decoding of variable length codes [25]. Hence, in contrast to other dimming control techniques, the unary code lends itself to iterative decoding by exchanging soft extrinsic information with the channel decoder. Furthermore, the complexity of MPPM encoding is of the order of $O\left(n^{2}\right)$ [8], while the unary encoder has a low encoding complexity, since it invokes a simple encoding procedure, which does not impose any computational complexity or memory requirements.

It is pertinent to mention here that the unary code probabilistically maintains the desired dimming level. Therefore, it may exhibit deviations from the target dimming value. However, if these fluctuations are within the Maximum Flickering Time Period (MFTP), which is around $5 \mathrm{~ms}$, then they are imperceptible by the human eye [26] - hence, achieving the 'flicker-free' dimming. For the sake of evaluating the impact of these fluctuations, in Fig. 4 we plot the Probability Density Function (PDF) of the dimming value observed at the output of the 8-level unary code, which is designed for achieving a dimming value of $78 \%$. We have averaged the dimming value over $N$ bits, where $N=1000$ and $N=5000$ in Fig. 4 . Explicitly, $N$ must meet the MFTP criterion. This implies that if the data rate is $D \mathrm{kbps}$, then $N \leq 5 \times D$, because any fluctuations within the MFTP of $5 \mathrm{~ms}$ remain imperceptible. As demonstrated in Fig. 4, increasing the value of $N$ from 1000 to 5000 significantly reduces the deviation from the target dimming level of $78 \%$. Since the data rates of VLC systems are of the order of several Megabits per second, or even Gigabits per second, $N$ can be potentially much higher than 5000 . Hence, the fluctuations in the unary coded output are not significant and can be safely ignored.

\section{UNARY-CODED ITERATIVE VLC SYSTEM}

Fig. 5 shows the schematic of the proposed unary-coded iterative VLC system, which consists of an outer FEC code and an inner unary code. Please note that a scrambler or unity-rate code [19] can be invoked before the unary code, if the input to the unary code is not strictly equiprobable.

At the transmitter, the FEC encoder of Fig. 5 encodes the intended information $x_{1}$, yielding the FEC-encoded bits $\bar{x}_{1}$. The encoded bits $\bar{x}_{1}$ are interleaved $(\pi)$ for generating the permuted sequence $x_{2}$, which is fed to the unary encoder. Recall that we intend to invoke the symbol-level trellis of [24] 


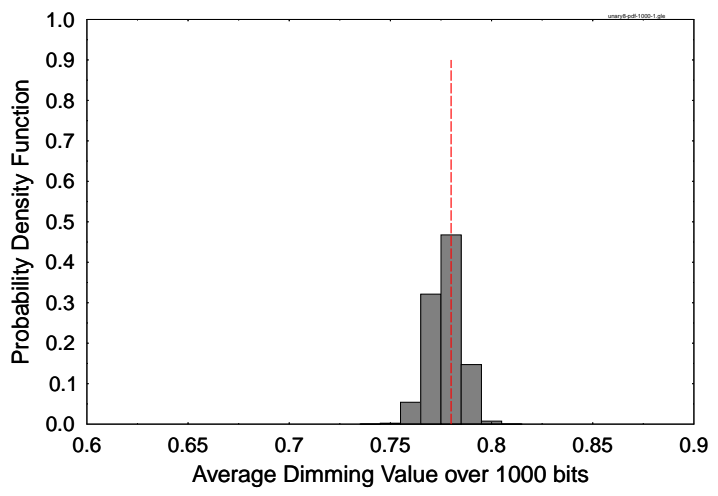

(a)

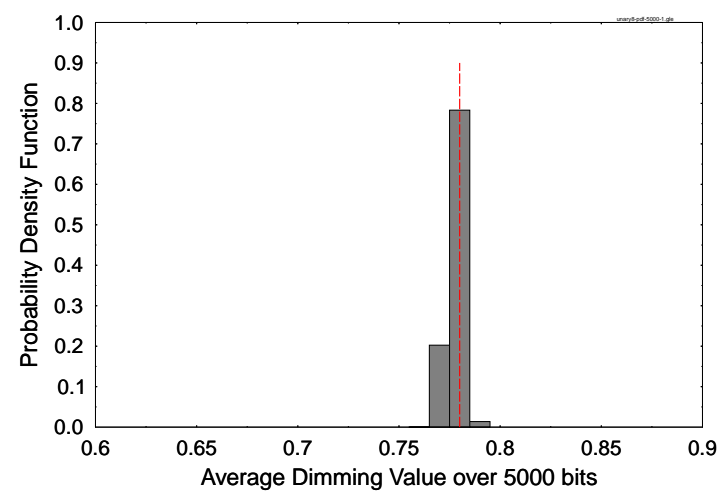

(b)

Fig. 4: Probability density function of the average dimming value at the output of the 8-level unary code of Table $\Pi$ Dashed-line shows the target dimming value of $\gamma_{u}=0.78$.

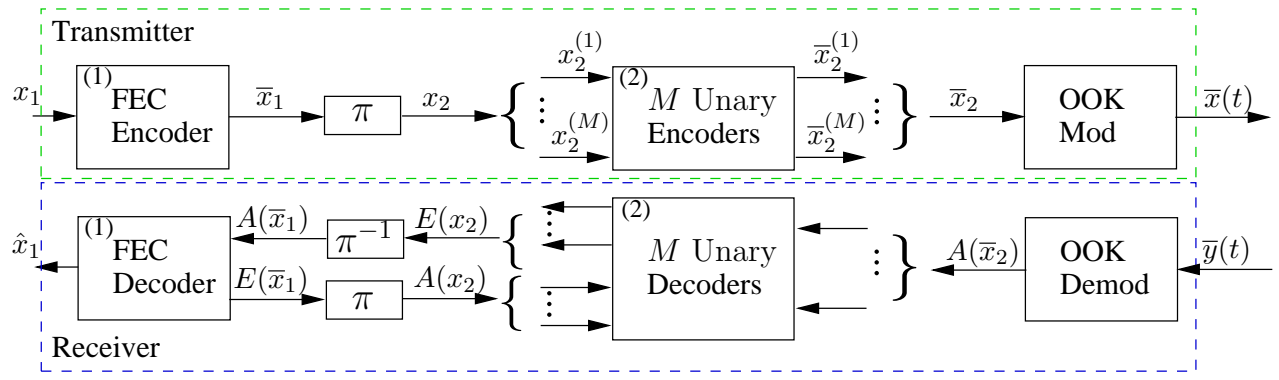

Fig. 5: Schematic of a unary-coded iterative VLC system. The a-priori and extrinsic LLRs, are denoted by $A($.$) and E(.),$ respectively.

for decoding the unary encoded bits. Since the complexity of symbol-level trellis increases upon increasing the frame length, we decompose the bit stream $x_{2}$ into $M$ sub-frames of $J$ symbols and encode each sub-frame separately by a unary encoder, which processes an equiprobable input to yield a $\gamma_{u}$-biased output, hence ensuring a dimming level of $\gamma_{u}$. Furthermore, since the unary codewords have variable length, each encoded sub-frame consists of different number of bits. For the sake of facilitating symbol-level trellis decoding, the number of encoded bits in each sub-frame also has to be reliably conveyed to the receiver. Explicitly, the symbollevel trellis is terminated according to both the number of encoded symbols as well as the number of encoded bits in each sub-frame. Hence, the length $J$ of sub-frames must be judiciously chosen to strike a reasonable trade-off between the decoding complexity and the amount of side-information transmitted. The resultant $M \gamma_{u}$-biased encoded sub-frames are concatenated and subsequently OOK modulated into $\bar{x}(t)$, which is emitted as a VLC signal.

At the receiver, the transmitted signal is detected with the aid of a photodetector, which transforms the received optical pulse into an electronic signal. The detected signal $\bar{y}(t)$ is processed by the OOK demodulator of Fig. 5, yielding the discrete signal $\bar{y}_{2}=\bar{x}_{2}+n$. Here $n$ represents the AWGN having a variance of $\sigma^{2}$. Next, the OOK demodulator computes the corresponding a-priori Logarithmic-Likelihood Ratios (LLRs) $A\left(\bar{x}_{2}\right)$, as follows:

$$
\begin{aligned}
A\left(\bar{x}_{2}\right) & \triangleq \ln \left(\frac{P\left(\bar{x}_{2}=1 \mid \bar{y}_{2}\right)}{P\left(\bar{x}_{2}=0 \mid \bar{y}_{2}\right)}\right) \\
& =\ln \left(\frac{P\left(\bar{y}_{2} \mid \bar{x}_{2}=1\right) P\left(\bar{x}_{2}=1\right)}{P\left(\bar{y}_{2} \mid \bar{x}_{2}=0\right) P\left(\bar{x}_{2}=0\right)}\right)
\end{aligned}
$$

where we have:

$$
P\left(\bar{y}_{2} \mid \bar{x}_{2}=a\right)=\frac{1}{2 \pi \sigma^{2}} e^{-\frac{\left|a-\bar{y}_{2}\right|^{2}}{2 \sigma^{2}}},
$$

while $P\left(\bar{x}_{2}=1\right)=\gamma_{u}$. Therefore, Eq. (7) is equivalent to:

$$
\begin{aligned}
A\left(\bar{x}_{2}\right) & =-\frac{1}{2 \sigma^{2}}\left(\left|1-\bar{y}_{2}\right|^{2}-\left|\bar{y}_{2}\right|^{2}\right)+\ln \left(\frac{\gamma_{u}}{1-\gamma_{u}}\right) \\
& =-\frac{1}{2 \sigma^{2}}\left(1-2 \bar{y}_{2}\right)+\ln \left(\frac{\gamma_{u}}{1-\gamma_{u}}\right) .
\end{aligned}
$$

It may be observed here that the second term of Eq. (9) reduces to zero, when the dimming level is 0.5. The LLRs $A\left(\bar{x}_{2}\right)$ of Eq. (9) are exchanged by the iterative decoding 
process between the inner and outer Soft-In Soft-Out (SISO) decoders, as shown in Fig. 5. Explicitly, analogous to the $M$ unary encoders at the transmitter, $M$ unary SISO decoders are invoked at the receiver. Consequently, the LLRs $A\left(\bar{x}_{2}\right)$ are partitioned into $M$ sub-frames based on the side information received about the number of encoded bits in each sub-frame. The $M$ sub-frames are fed separately to $M$ unary SISO decoders and the resultant extrinsic information is concatenated, as shown in Fig. 5. Finally, the SISO decoders of the inner (unary) and the outer (FEC) components engage in iterative decoding, hence iteratively exchanging extrinsic information, as shown in Fig. 5] Please note that the notations $A($.$) and$ $E($.$) represent the a-priori and extrinsic LLRs, respectively.$

\section{EXIT CHART BASED CAPACITY ANALYSIS}

EXtrinsic Information Transfer (EXIT) chart [17], [20], [27] constitute a powerful semi-analytical tool, which helps in visualizing the convergence behaviour of an iterative decoding process. Explicitly, the EXIT chart visualizes the flow of average Mutual Information (MI) between the concatenated components of an iterative decoder. This is achieved with the aid of the inner and outer decoders' EXIT curves, which are constructed by modeling the associated a priori information, $A\left(x_{2}\right)$ and $A\left(\bar{x}_{1}\right)$ respectively, using an independent Gaussian distribution for a range of $I_{A}(z) \in[0,1]$, where $I_{A}(z)$ denotes the average MI between $z$ and $A(z)$, while $z \in\left\{x_{2}, \bar{x}_{1}\right\}$. The output extrinsic MI, $I_{E}\left(x_{2}\right)$ and $I_{E}\left(\bar{x}_{1}\right)$ of the inner and outer decoders respectively, are then calculated as follows [17], [20], [27]:

$$
I_{E}(z)=1-\mathrm{E}\left(\mathrm{H}_{b}\left(\frac{1}{1+e^{E(z)}}\right)\right),
$$

where $\mathrm{E}($.$) denotes the expectation operator (time-average),$ while $\mathrm{H}_{b}($.$) is the binary entropy function. It is important to$ note here that the maximum value of MI is 1 , because $x_{2}$ and $\bar{x}_{1}$ consist of equiprobable bits. The pair of MI characteristics defined by $I_{A}\left(x_{2}\right)$ and $I_{E}\left(x_{2}\right)$ constitute the inner decoder's EXIT curve having the transfer function $T_{2}$, while $I_{A}\left(\bar{x}_{1}\right)$ and $I_{E}\left(\bar{x}_{1}\right)$ yield the outer decoder's EXIT curve having the transfer function $T_{1}$, which may be formulated as:

$$
I_{E}\left(x_{2}\right)=T_{2}\left[I_{A}\left(x_{2}\right), \frac{E_{c}}{N_{0}}\right], \quad I_{E}\left(\bar{x}_{1}\right)=T_{1}\left[I_{A}\left(\bar{x}_{1}\right)\right] .
$$

It may be observed in Eq. (11) that $T_{2}$ is a function of the channel SNR $E_{c} / N_{0}$, since the inner decoder relies on the received information of Eq. (9). Furthermore, the average signal power $E_{c}$ is equivalent to the dimming level $\gamma_{u}$. Finally, both the inner and the inverted outer EXIT curves are plotted in the same graph, so that the flow of information between the two concatenated decoders can be characterized by a staircase-shaped decoding trajectory.

We next exploit the area property [17], [20] of EXIT charts for quantifying the maximum throughput achievable by the proposed unary-coded dimming schemes over an AWGN channel, which we refer to as the 'unary-constrained AWGN capacity'. Explicitly, the area $\mathcal{A}_{2}$ under the inner decoder's EXIT curve is equivalent to [20]:

$$
\mathcal{A}_{2}=\frac{C_{\mathrm{u}}}{R_{u}}
$$

where $R_{u}$ is the coding rate of the inner component, which in our system is the unary code as shown in Fig. 5, while $C_{\mathrm{u}}$ characterizes the unary-constrained AWGN capacity. Furthermore, we have $C_{\mathrm{u}}<C$, where $C$ is the unconstrained AWGN channel capacity (also known as the Continuous-input Continuous-output Memoryless Channel (CCMC) capacity) for a $\gamma_{u}$-biased input. More specifically, the unconstrained AWGN channel capacity $C$ is formulated as:

$$
\begin{aligned}
C & =\mathrm{H}\left(\bar{x}_{2}\right)-\mathrm{H}\left(\bar{x}_{2} \mid \bar{y}_{2}\right) \\
& =\mathrm{H}_{b}\left(\gamma_{u}\right)-\mathrm{H}\left(\bar{x}_{2} \mid \bar{y}_{2}\right),
\end{aligned}
$$

where the entropy $\mathrm{H}\left(\bar{x}_{2} \mid \bar{y}_{2}\right)$ may be computed using the LLRs of Eq. (9). Finally, $\left(C-C_{\mathrm{u}}\right)$ quantifies the inherent capacity (or throughput) loss, relative to the unconstrained AWGN channel capacity, incurred by using a unary code for dimming control.

Fig. 6 compares the unary-constrained AWGN capacity $C_{\mathrm{u}}$ of the proposed unary-coded schemes, namely the 4-level unary and the 8-level unary schemes, with the corresponding unconstrained AWGN channel capacity $C$ as well as the equivalent compensation time dimming scheme (CT) of Section II-A We have used a sub-frame length of $J=100$ symbols for generating the associated inner EXIT curves. Recall from Fig. 2] that unary-coded schemes are capable of achieving a higher throughput than compensation time dimming for $\gamma<0.4$ and $\gamma>0.6$. We may observe in Fig. 6a that unary code offers a slightly higher throughput than compensation time dimming at low SNRs, even when $\gamma=0.6$ (or equivalently $\gamma=0.4$ ). Furthermore, it can be seen in Fig. 6 that the capacity loss reduces upon decreasing the SNR and ultimately disappears. This is true for both the unary codes as well as for compensation time dimming. However, it occurs earlier for the unary schemes. We may also notice that the capacity curve of the 4-level unary scheme ultimately crosses over the unconstrained AWGN capacity. This is due to trellis termination. We observed that the impact of termination tends to disappear, when longer sub-frame lengths are invoked.

EXIT charts may be invoked for designing unary-coded iterative VLC systems operating close to the unary-constrained capacity of Fig. 6 More specifically, the area under the inverted outer decoder's EXIT curve is equivalent to the coding rate of the outer component [20]. Therefore, having an open tunnel between the inner decoder and the inverted outer decoder EXIT curve, which is referred to as an EXIT-chart tunnel, implies that the resultant system throughput is lower than the unary-constrained AWGN capacity $C_{\mathrm{u}}$, which in turn guarantees achieving decoding convergence to a vanishingly low BER. Furthermore, the area of the EXIT-chart tunnel quantifies the rate loss with respect to the unary-constrained AWGN capacity $C_{\mathrm{u}}$. Hence, a capacity approaching design aims for creating a narrow, but marginally open EXIT-chart tunnel at the lowest possible SNR. 


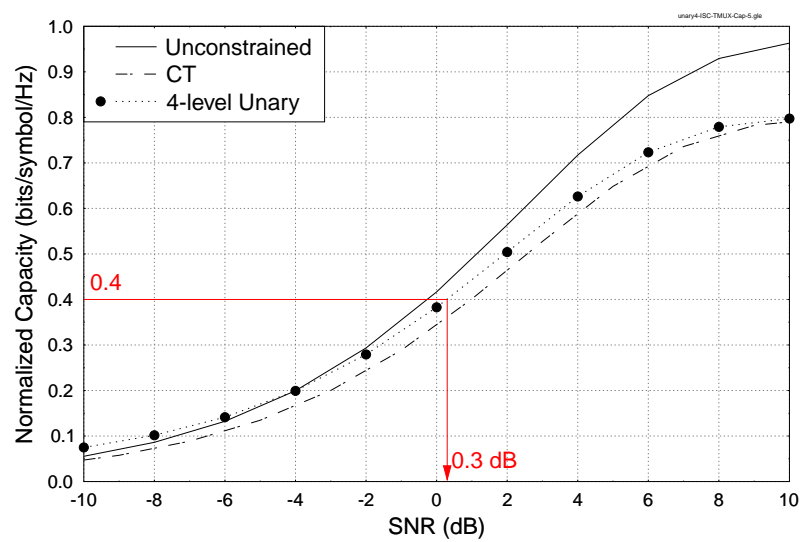

(a) 4-level unary $\left(\gamma_{\mathrm{u}}=0.6, R_{\mathrm{u}}=0.8\right)$.

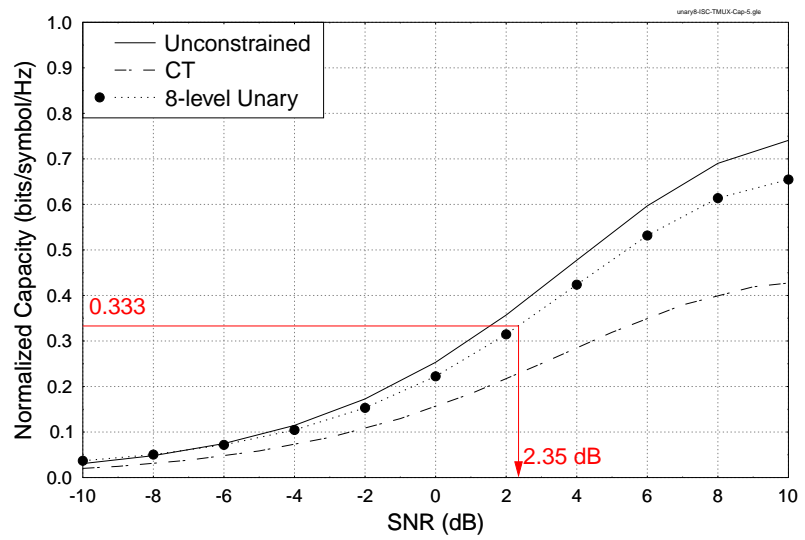

(b) 8-level unary $\left(\gamma_{\mathrm{u}}=0.78, R_{\mathrm{u}}=0.667\right)$.

Fig. 6: Unary-constrained capacity over an AWGN channel.

\section{DESIGN EXAMPLES: RESUlts AND Discussions}

For the sake of evaluating the performance of the proposed unary-coded iterative VLC system, in this section we design coding schemes for dimming levels of $\gamma=0.6$ and $\gamma=0.78$. More specifically, our design examples rely on the 4-level and 8-level unary codes, while a 1/2-rate FEC code constitutes the outer component. Hence, the resultant systems have a dimming level of $\gamma=0.6$ and $\gamma=0.78$, respectively, and normalized throughputs of $(0.8 \times 0.5)=0.4$ and $(0.667 \times 0.5)=0.333$, respectively. According to the unary-constrained AWGN capacity curves of Fig. 6, the corresponding SNR limits for reliable transmission are about $0.3 \mathrm{~dB}$ and $2.35 \mathrm{~dB}$, respectively. Please note that we have used an interleaver of length 10,000 bits for the 4-level unary schemes, while a 12,000-bit long interleaver is used for the 8 -level unary-coded schemes. Furthermore, a sub-frame of length $J=100$ symbols is used for partitioning the frames for unary encoding/decoding. We benchmark the performance of the proposed iterative designs against non-iterative schemes, which invoke 1/2-rate outer FEC codes in conjunction with compensation time dimming. The resultant compensation time dimming based VLC system has a normalized throughput of $(0.8 \times 0.5)=0.4$ at $\gamma=0.6$ and $(0.44 \times 0.5)=0.22$ at $\gamma=0.78$. Hence, the 8 -level unary schemes offer 1.5 times higher throughput than the compensation time dimming based benchmarks for $\gamma=0.78$. It is pertinent to mention here that our unary-coded schemes of Table [II] offer dimming control over a wide range of dimming values. However, the overall system throughput degrades upon increasing (or equivalently decreasing) the target dimming value, as previously seen in Fig. 2. Nevertheless, the throughput of unary-coded schemes is almost always closest to the ISC limit. Specifically, the unary-coded schemes offer higher throughput than the classic compensation time dimming approach for $\gamma>0.6$ (or equivalently $\gamma<0.4$ ) and the achievable throughput gain increases upon increasing the required dimming level.
Design Example I: As our first example, we analyze 4level and 8-level unary-coded iterative VLC systems, which invoke a unit memory $1 / 2$-rate convolutional code having the generators $\left(g_{1}, g_{2}\right)=(3,2)_{8}$ as the outer component.

Fig. 7 shows the EXIT curves of the 4-level and 8-level unary-coded schemes. It may be observed in Fig. 7 that the inner EXIT curves of the unary codes terminate at the $(1,1)$ point of perfect convergence; hence, facilitating convergence to an infinitesimally low BER, provided that the EXIT-chart tunnel is open and a sufficiently long interleaver is used. Explicitly, a marginally open tunnel exits at SNR $=2 \mathrm{~dB}$ and SNR $=4 \mathrm{~dB}$ in Fig. 7a and Fig. 7b, respectively, which marks the convergence threshold of these schemes. These convergence thresholds are within $1.7 \mathrm{~dB}$ and $1.65 \mathrm{~dB}$ of the corresponding unary-constrained AWGN capacities. If the channel SNR drops below the convergence threshold, the inner and outer decoders' EXIT curves will crossover, thereby closing the EXIT-chart tunnel and hence failing to converge to a low BER. Fig. 7a and Fig. 7b also show two decoding trajectories superimposed on the EXIT chart at an SNR of $3 \mathrm{~dB}$ and $5 \mathrm{~dB}$, respectively. The decoding trajectories are in line with the associated EXIT curves, reaching the $(1,1)$-point of perfect convergence.

Fig. 8 records the resultant BER performance after 5, 10 and 15 iterations. The Turbo-cliff emerges around $2.2 \mathrm{~dB}$ and $4.2 \mathrm{~dB}$ for the 4-level and 8-level schemes, respectively, which is close to our EXIT chart predictions. We have further benchmarked the performance of the unary-coded schemes against non-iterative schemes employing the same convolutional code, but in conjunction with compensation time dimming (labeled 'CT'). Quantitatively, at a BER of $10^{-5}$, the 4-level unarycoded design $(\gamma=0.6)$ offers around $6 \mathrm{~dB}$ gain relative to the compensation time dimming scheme after 10 iterations, while the 8-level unary-coded design $(\gamma=0.78)$ yields approximately $5.1 \mathrm{~dB}$ gain.

We may observe in Fig. 8 that despite their benefits, the 

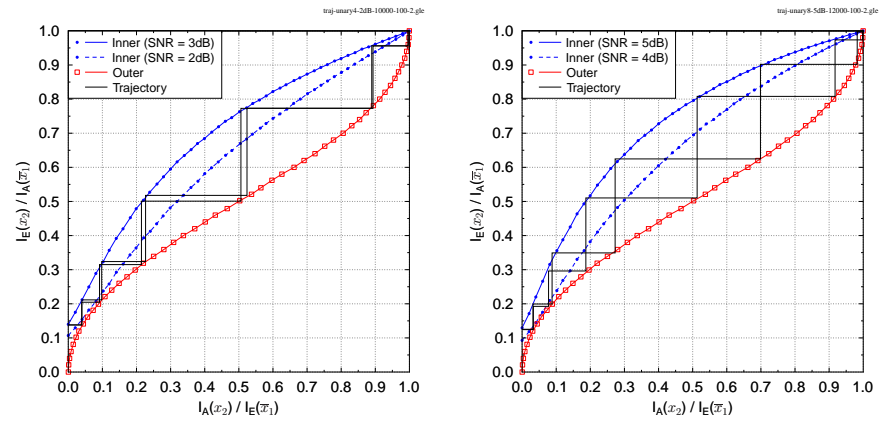

(a) 4-level unary $\left(\gamma_{\mathrm{u}}=0.6, R_{\mathrm{u}}=\right.$ (b) 8-level unary $\left(\gamma_{\mathrm{u}}=0.78\right.$, $0.8)$.

$\left.R_{\mathrm{u}}=0.667\right)$.

Fig. 7: EXIT curves of unary-coded VLC systems, when a memory-1 1/2-rate convolutional code having the octal generators of $\left(g_{1}, g_{2}\right)=(3,2)_{8}$ is used as the outer component. The stair-case-shaped decoding trajectories are plotted at $S N R=3 d B$ and $S N R=5 d B$ for the 4-level and 8-level unary schemes, respectively.

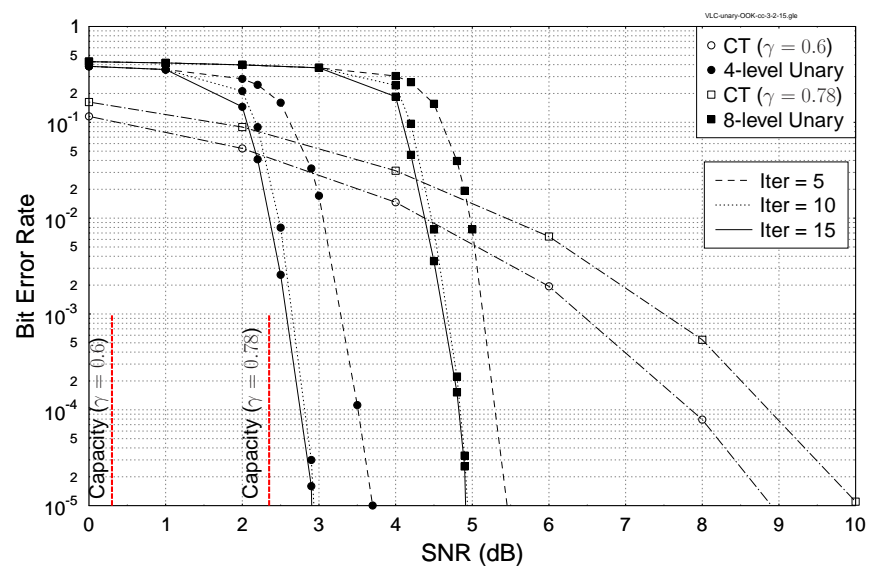

Fig. 8: BER performance of the 4-level and 8-level unarycoded schemes, when a memory-1 1/2-rate convolutional code having the octal generators of $\left(g_{1}, g_{2}\right)=(3,2)_{8}$ is used as the outer component. The performance is benchmarked both against the compensation time dimming approach (CT) as well as the unary-constrained AWGN capacity. Iterleavers of length 10,000 and 12,000 were used for the 4-level and 8-level schemes, respectively.

performance of the systems is not so close to the achievable unary-constrained AWGN capacity. This discrepancy is commensurate with the wide area of the EXIT-chart tunnel in Fig. 7 A near-capacity design may be achieved by designing an outer code having a similar EXIT-curve shape to that of the inner code, which yields a marginally open EXIT-chart tunnel at an SNR arbitrarily close to the capacity limit. However, this in turn imposes an increased decoding complexity, since more decoding iterations are required to reach the $(1,1)$-point of perfect convergence, when the EXIT-chart tunnel is narrow.

Design Example II: For the sake of achieving a capacityapproaching unary-coded iterative VLC system, we invoke the 17-subcode IRCC of [21] as the outer component.

The IRCC of [21] consists of 17 subcodes $\mathcal{C}_{l}$, where $1 \leq l \leq$ 17 , each having a different coding rate. Explicitly, the subcodes have coding rates of $r_{l} \in\{0.1,0.15,0.2, \ldots, 0.85,0.9\}$, which cover the complete code-rate range spanning from 0.1 to 0.9 . This set of subcodes $\mathcal{C}_{l}$ is generated by using a memory- $41 / 2$ rate convolutional code as a mother code and constructing the remaining 16 subcodes by either puncturing or using additional generator polynomials. The input information bits of IRCC are divided into 17 sub-frames corresponding to the 17 constituent subcodes, so that the $l$ th code $\mathcal{C}_{l}$, having the coding rate $r_{l}$, yields $\varrho_{l}$-fraction of the coded frame. The fraction $\varrho_{l}$ is referred to as the weighting coefficient of the $l$ th subcode, which has to satisfy the following constraints [21], [28]:

$$
\sum_{l=1}^{17} \varrho_{l}=1, R_{o}=\sum_{l=1}^{17} \varrho_{l} r_{l}, \varrho_{l} \in[0,1], \forall l .
$$

It can be expressed in matrix notation as follows:

$$
\begin{aligned}
{\left[\begin{array}{cccc}
1 & 1 & \ldots & 1 \\
r_{1} & r_{2} & \ldots & r_{17}
\end{array}\right]\left[\begin{array}{lll}
\varrho_{1} & \varrho_{2} \ldots & \varrho_{17}
\end{array}\right]^{T}=} & {\left[\begin{array}{c}
1 \\
R_{o}
\end{array}\right] } \\
\mathbf{C} \varrho & =\mathbf{d} .
\end{aligned}
$$

The inverted outer decoder's EXIT curves of the constituent IRCC subcodes are plotted in Fig. 9, while the overall transfer function of the IRCC is given by:

$$
I_{E}\left(\bar{x}_{1}\right)=T_{1}\left[I_{A}\left(\bar{x}_{1}\right)\right]=\sum_{l=1}^{17} \varrho_{l} T_{1, l}\left[I_{A}\left(\bar{x}_{1}\right)\right],
$$

where $T_{1, l}\left[I_{A}\left(\bar{x}_{1}\right)\right]$ is the transfer function of the $l^{\text {th }}$ subcode. The weighting coefficients $\varrho_{l}$ in Eq. (16) may be optimized using the curve matching algorithm of [21], [28] so that the EXIT-chart tunnel area is minimized at an SNR close to the capacity limit.

Fig. 10 shows the EXIT curves of the 4-level and 8-level unary-coded schemes, when a $1 / 2$-rate IRCC is employed as the outer component. The resultant convergence thresholds of the 4-level and 8-level schemes are $0.5 \mathrm{~dB}$ and $2.5 \mathrm{~dB}$, respectively. Hence, the IRCC aided schemes are capable of operating within $0.2 \mathrm{~dB}$ and $0.15 \mathrm{~dB}$ of the unary-constrained AWGN capacity. The resultant BER performance is recorded in Fig. 11 for 10, 20 and 30 iterations. We may observe in Fig. 11 that the performance of our 4-level unary-coded design $(\gamma=0.6)$ is within around $1.6 \mathrm{~dB}$ of the capacity limit at a BER of $10^{-5}$ and after 20 iterations, while that of the 8-level scheme $(\gamma=0.78)$ is around $1.3 \mathrm{~dB}$ away from the capacity. We have further compared the performance of our iterative design to the non-iterative compensation time dimming, when it is employed in conjunction with the strong memory-4 1/2-rate subcode of IRCC. Again, our iterative designs significantly outperform their compensation time dimming based counterparts, albeit 


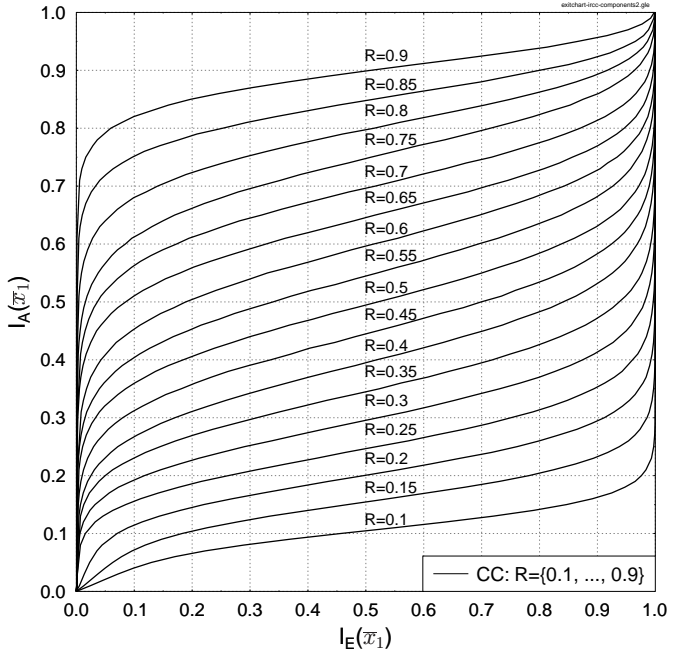

Fig. 9: Inverted outer EXIT curves (normalized) of the 17 subcodes of IRCC.
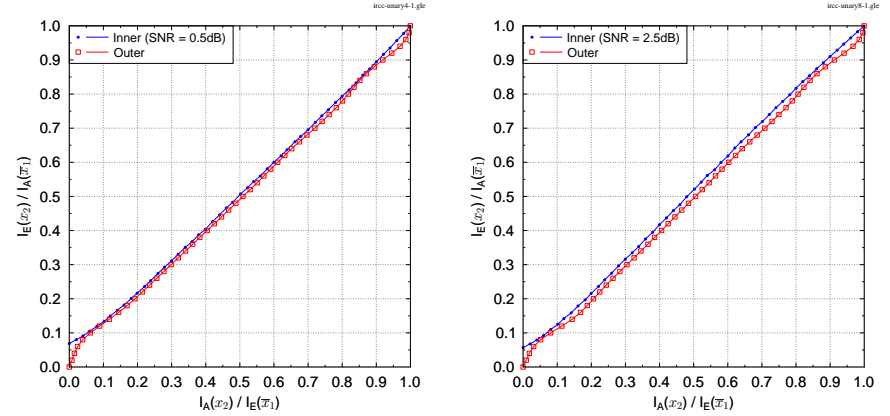

(a) 4-level unary $\left(\gamma_{\mathrm{u}}=0.6, R_{\mathrm{u}}=\right.$ (b) 8-level unary $\left(\gamma_{\mathrm{u}}=0.78\right.$, $0.8)$.

$\left.R_{\mathrm{u}}=0.667\right)$.

Fig. 10: EXIT curves of unary-coded VLC systems, when $1 / 2$ rate IRCC is used as the outer component.

at the cost of an increased decoding complexity. Explicitly, at a BER of $10^{-5}$ and after 20 decoding iterations, the unarycoded design yields a gain of around $4.4 \mathrm{~dB}$ and $3.6 \mathrm{~dB}$, when considering dimming levels of $\gamma=0.6$ and $\gamma=0.78$, respectively.

\section{CONCLUSIONS}

In this paper, we have conceived a unary-coded dimming control scheme for OOK-modulated VLC systems. The proposed dimming scheme provides joint throughput and dimming control, while also facilitating the implementation of iterative decoding. It is demonstrated that the unary-coded dimming offers a higher throughput than the conventional compensation time dimming as well as the MPPM based schemes and ultimately approaches the theoretical limit. We further quantified

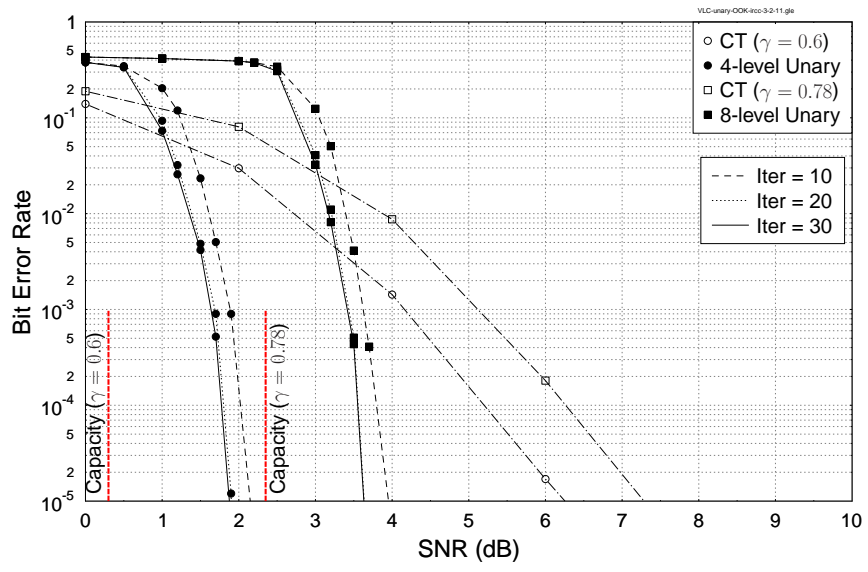

Fig. 11: BER performance of the 4-level and 8-level unarycoded schemes, when a $1 / 2$-rate IRCC is used as the outer component. The performance is benchmarked against the compensation time dimming approach (CT) as well as the unaryconstrained AWGN capacity. Iterleavers of length 10, 000 and 12,000 bits were used for the 4-level and 8-level schemes, respectively.

the unary-constrained AWGN capacity and designed iterative unary-FEC schemes with the aid of EXIT charts. This is the first instantiation of iteratively decoded joint dimming-FEC coding schemes. Our results demonstrate that unary-coded iterative schemes significantly outperform their compensation time dimming based counterparts. Quantitatively, our 4-level unary-coded designs $(\gamma=0.6)$ yield a gain of about 5 to $6 \mathrm{~dB}$, while the 8 -level schemes $(\gamma=0.78)$ provide a gain of about 3 to $4 \mathrm{~dB}$ in addition to offering a 1.5 times higher throughput. Furthermore, the IRCC-based designs operate arbitrarily close to the unary-constrained AWGN capacity. However, this is achieved at the cost of an increased decoding complexity.

\section{REFERENCES}

[1] "IEEE standard for local and metropolitan area networks-part 15.7: Short-range wireless optical communication using visible light," IEEE Std 802.15.7-2011, pp. 1-309, Sept 2011.

[2] X. Li, R. Zhang, and L. Hanzo, "Cooperative load balancing in hybrid visible light communications and WiFi," IEEE Transactions on Communications, vol. 63, no. 4, pp. 1319-1329, April 2015.

[3] J. Jiang, R. Zhang, and L. Hanzo, "Analysis and design of threestage concatenated color-shift keying," IEEE Transactions on Vehicular Technology, vol. 64, no. 11, pp. 5126-5136, Nov 2015.

[4] C. Zhu, Y. Huo, J. Jiang, H. Sun, C. Dong, R. Zhang, and L. Hanzo, "Hierarchical colour-shift-keying aided layered video streaming for the visible light downlink," IEEE Access, vol. 4, pp. 3127-3152, 2016.

[5] F. Zafar, D. Karunatilaka, and R. Parthiban, "Dimming schemes for visible light communication: the state of research," IEEE Wireless Communications, vol. 22, no. 2, pp. 29-35, April 2015.

[6] J. Kwon, "Inverse source coding for dimming in visible light communications using NRZ-OOK on reliable links," IEEE Photonics Technology Letters, vol. 22, no. 19, pp. 1455-1457, Oct 2010. 
[7] K. Lee and H. Park, "Modulations for visible light communications with dimming control," IEEE Photonics Technology Letters, vol. 23 , no. 16, pp. 1136-1138, Aug 2011.

[8] A. B. Siddique and M. Tahir, "Joint rate-brightness control using variable rate MPPM for LED based visible light communication systems," IEEE Transactions on Wireless Communications, vol. 12, no. 9, pp. 4604-4611, September 2013.

[9] H. Park and J. R. Barry, "Trellis-coded multiple-pulse-position modulation for wireless infrared communications," IEEE Transactions on Communications, vol. 52, no. 4, pp. 643-651, April 2004.

[10] F. Xu, M. A. Khalighi, and S. Bourennane, "Coded PPM and multipulse PPM and iterative detection for free-space optical links," IEEE/OSA Journal of Optical Communications and Networking, vol. 1, no. 5, pp. 404-415, Oct 2009.

[11] T. T. Nguyen and L. Lampe, "Coded multipulse pulse-position modulation for free-space optical communications," IEEE Transactions on Communications, vol. 58, no. 4, pp. 1036-1041, April 2010.

[12] A. B. Siddique and M. Tahir, "Joint error-brightness control coding for LED based VLC link," in 2014 IEEE Wireless Communications and Networking Conference (WCNC), April 2014, pp. 400-404.

[13] S. Kim and S.-Y. Jung, "Novel FEC coding scheme for dimmable visible light communication based on the modified Reed-Muller codes," IEEE Photonics Technology Letters, vol. 23, no. 20, pp. 1514-1516, Oct 2011.

[14] S. H. Lee and J. K. Kwon, "Turbo code-based error correction scheme for dimmable visible light communication systems," IEEE Photonics Technology Letters, vol. 24, no. 17, pp. 1463-1465, Sept 2012.

[15] S. Kim and S. Y. Jung, "Modified Reed-Muller coding scheme made from the bent function for dimmable visible light communications," IEEE Photonics Technology Letters, vol. 25, no. 1, pp. 11-13, Jan 2013.

[16] J. Kim and H. Park, "A coding scheme for visible light communication with wide dimming range," IEEE Photonics Technology Letters, vol. 26, no. 5, pp. 465-468, March 2014.

[17] L. Hanzo, T. H. Liew, B. L. Yeap, R. Y. S. Tee and S. X. Ng, Turbo Coding, Turbo Equalisation and Space-Time Coding: EXIT-Chart-Aided Near-Capacity Designs for Wireless Channels, 2nd Edition. New York, USA: John Wiley IEEE Press, March 2011.

[18] X. Lu and J. L. Tiffany, "Achieving FEC and RLL for VLC: A concatenated convolutional-Miller coding mechanism," IEEE Photonics Technology Letters, vol. 28, no. 9, pp. 1030-1033, May 2016.

[19] Z. Babar, H. V. Nguyen, P. Botsinis, D. Alanis, D. Chandra, S. X. Ng, and L. Hanzo, "Unity-rate codes maximize the normalized throughput of ON-OFF keying visible light communication," IEEE Photonics Technology Letters, vol. 29, no. 3, pp. 291-294, Feb 2017.

[20] A. Ashikhmin, G. Kramer, and S. ten Brink, "Extrinsic information transfer functions: model and erasure channel properties," IEEE Transactions on Information Theory, vol. 50, no. 11, pp. 2657- 2673, November 2004.

[21] M. Tüchler, "Design of serially concatenated systems depending on the block length," IEEE Transactions on Communications, vol. 52, no. 2, pp. 209-218, February 2004.

[22] R. Gallager and D. van Voorhis, "Optimal source codes for geometrically distributed integer alphabets (corresp.)," IEEE Transactions on Information Theory, vol. 21, no. 2, pp. 228-230, Mar 1975.

[23] R. G. Maunder, W. Zhang, T. Wang, and L. Hanzo, "A unary error correction code for the near-capacity joint source and channel coding of symbol values from an infinite set," IEEE Transactions on Communications, vol. 61, no. 5, pp. 1977-1987, May 2013.

[24] R. Bauer and J. Hagenauer, "Symbol-by-symbol map decoding of variable length codes," in in Proc. 3. ITG Conf. on Source and Channel Coding, 2000, pp. 111-116.

[25] L. Hanzo, R. Maunder, J. Wang, and L. Yang, Near-Capacity Variable-Length Coding: Regular and EXIT-Chart-Aided Irregular Designs, ser. Wiley - IEEE. Wiley, 2010. [Online]. Available: https://books.google.com.pk/books?id=naxtTIvhWxAC
[26] S. M. Berman, D. S. Greenhouse, I. L. Bailey, R. D. Clear, and T. W. Raasch, "Human electroretinogram responses to video displays, fluorescent lighting, and other high frequency sources." Optometry \& Vision Science, vol. 68, no. 8, pp. 645-662, 1991.

[27] M. El-Hajjar and L. Hanzo, "EXIT charts for system design and analysis," IEEE Communications Surveys Tutorials, pp. 1-27, 2013.

[28] M. Tüchler and J. Hagenauer, "EXIT charts of irregular codes," in Proceedings of Conference on Information Science and Systems, Princeton University, 20-22 March 2002, pp. 465-490. 